Reprinted from Journal of the Frankitn Institute, Vol. 276, No. 2, August, 1963

Printed in U. S. A.

\title{
THEORY OF STABILITY OF MULTILAYERED CONTINUA IN FINITE ANISOTROPIC ELASTICITY
}

\author{
BY
}

\author{
M. A. BIOT
}

\begin{abstract}
The writer's general equations for the mechanics of continua under initial stress are applied to the formulation of a rigorous theory of stability of multilayered elastic media in a state of finite initial strain. The medium is assumed incompressible. It is either isotropic or anisotropic. The problem is analyzed in the context of the writer's earlier discussions showing the existence of internal and interfacial instability. The results provide a rigorous solution of the problem of buckling of sandwich plates. Recurrence equations are derived for the interfacial displacements. It is shown that they are equivalent to a variational principle expressed in terms of these displacements. A matrix multiplication procedure is also developed for automatic computing of critical values when a large number of layers is involved.
\end{abstract}

\section{INTRODUCTION}

The theory of stability of a continuum under initial stress was developed by the writer more than twenty years ago $(1,2,3) .^{2}$ More recently it was applied to problems of stability of elastic and viscoelastic media. Stability problems have been solved and discussed for the following cases: the isotropic homogencous and nonhomogeneous half space of elastic and viscoelastic properties $(4,5)$ the embedded layer with elastic and viscoelastic isotropy $(4,6)$, and the surface instability and the buckling of a thick slab of rubber in finite initial strain $(\mathbf{7}, \mathbf{8})$. The analysis was restricted to materials which retain isotropy under initial stress for incremental plane strain. Another series of papers introduced elastic anisotropy, whether induced by the initial finite strain or inherent originally in the stress-free material. The phenomenon of internal instability implicit in the writer's earlier paper on wave propagation (9) was given a detailed discussion and analysis (10). Further developments included the solutions for the problems of surface and interfacial stability under conditions of anisotropy in finite elasticity $(11,12)$.

'These results provided the essential foundation for the treatment of the more complex problems analyzed in the present paper. In particular, it was necessary to clarify the nature of internal instability as well as surface and interfacial instability. This is quite analogous to the analysis of wave propagation in layered media, where a thorough under-

${ }^{1}$ Shell Development Company, New York, N. Y.

${ }^{2}$ The boldface numbers in parentheses refer to the references appended to this paper. 
standing of the behavior of body waves, Rayleigh and Stoneley waves is essential before any sound treatment of more complex structures can be undertaken.

This paper analyzes the stability of an elastic medium made of a superposition of layers of finite or infinite thickness. The medium is assumed to be incompressible. The assumption of incompressibility does not affect essentially the generality of the results and introduces considerable simplification in the algebra.

The theory applies to an elastic continuum in a state of homogeneous finite strain. The state of initial stress, which may be different in each layer, has principal directions which are the same in all layers, one of these being perpendicular to the layers. The material in each layer may be orthotropic with the same planes of symmetry as the initial stress or it may be isotropic in the original stress-free state. In the latter case the initial stress will induce an elastic anisotropy with the same planes of symmetry. The incremental elastic coefficients for the case of a medium isotropic in finite strain were derived recently $(10,13)$.

Some basic results derived in previous work are briefly outlined in Section 2. They refer to general solutions for the anisotropic medium under initial stress and a discussion of the elastic coefficients for laminated materials and for an elastic continuum which is isotropic in finite strain.

These results are applied in Section 3 to a single anisotropic plate under initial stress. Equations are derived for the surface displacements under normal and tangential forces. Limiting cases are discussed in Section 4. These include the case of infinite thickness and the classical degenerate case of an isotropic medium with vanishing initial stress.

The multilayered system is treated in Section 5 . The results for the single plate are used to derive a general formulation of the stability equations for multilayered media. Recurrence and matrix equations are obtained which are well suited for numerical solution with automatic computers. The matrix multiplication procedure is suggested by a method proposed by Haskell for the analogous case of wave propagation. The recurrence equations also lead to a variational principle expressed in terms of interfacial displacements as arbitrary variables.

\section{FUNDAMENTAL EQUATIONS AND GENERAL SOLUTIONS}

Consider the two-dimensional deformation of an anisotropic continuum under initial stress. The deformation is in the $x, y$ plane and the initial stress is a uniform compression $P$ parallel with the $x$ direction. Earlier work has shown that the incremental stresses $s_{11}, s_{22}, s_{12}$ satisfy the equilibrium conditions 


$$
\begin{aligned}
& \frac{\partial s_{11}}{\partial x}+\frac{\partial s_{12}}{\partial y}-P \frac{\partial \omega}{\partial y}=0 \\
& \frac{\partial s_{12}}{\partial x}+\frac{\partial s_{22}}{\partial y}-P \frac{\partial \omega}{\partial x}=0
\end{aligned}
$$

The local rotation $\omega$ is

$$
\omega=\frac{1}{2}\left(\frac{\partial v}{\partial x}-\frac{\partial u}{\partial y}\right)
$$

where $u$ and $v$ are the components of displacement. The incremental stress components are referred to rotated axes.

The general equations (A1) for plane strain are written out in the Appendix. Equations 1 are obtained by putting

in Eqs. (A1).

$$
\begin{aligned}
X & =Y=0 \\
S_{22} & =S_{12}=0 \\
S_{11} & =-P
\end{aligned}
$$

A principal stress $S_{33}$ may or may not be present in a direction perpendicular to the $x, y$ plane. This component of the initial stress may be disregarded since it does not appear explicitly in the plane strain theory.

The medium is assumed to be incompressible and orthotropic with the directions of symmetry parallel with the coordinate axes. For an elastic medium the incremental stress-strain relations are

$$
\begin{aligned}
s_{11}-s & =2 N e_{x x} \\
s_{22}-s & =2 N e_{y y} \\
s_{12} & =2 Q e_{x y} .
\end{aligned}
$$

The left-hand side of these equations represents the incremental stress deviator in two dimensions. It is not the same as the three-dimensional deviator. The strain components are

$$
e_{x x}=\frac{\partial u}{\partial x} \quad e_{y y}=\frac{\partial v}{\partial y} \quad e_{x y}=\frac{1}{2}\left(\frac{\partial v}{\partial x}+\frac{\partial u}{\partial y}\right) .
$$

The condition of incompressibility must be added:

$$
e_{x x}+e_{y y}=0 \text {. }
$$

The stress-strain relations (4) have been written with elastic coefficients $N$ and $Q$. The case of an orthotropic viscoelastic medium is 
formally identical with the elastic case. By the correspondence principle introduced by this writer the elastic coefficients are replaced by operators $N^{*}$ and $Q^{*}$. The algebra is the same for both cases and may be carried out with the elastic coefficients. Results are readily extended to the viscoelastic medium by substituting the operators in the final results.

An important expression in the present problem is that of the incremental force acting on a deformed surface which is initially a plane perpendicular to the $y$ axis. The force is expressed per unit initial area before deformation. It is considered to act on the bottom half space as shown in Fig. 1. The $x$ and $y$ components of this force were derived
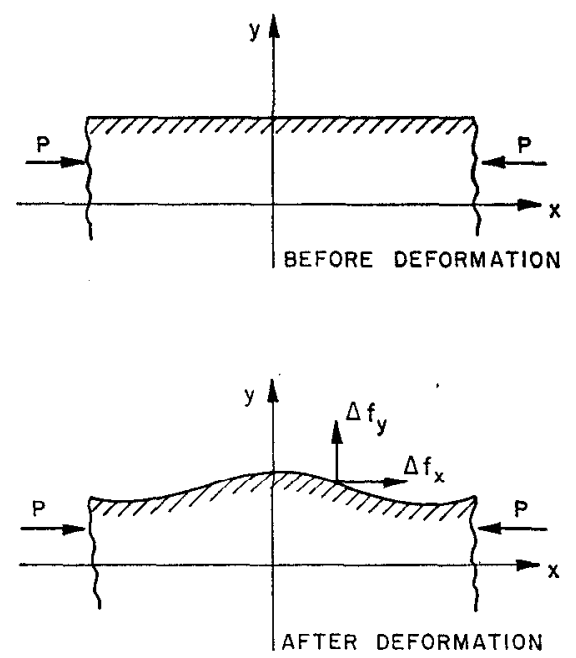

FIG. 1. Illustration of the incremental force components $\Delta f_{x}, \Delta f_{v}$.

in earlier work and may be found by applying Eqs. A2 of the Appendix. They are

$$
\begin{aligned}
& \Delta f_{x}=(2 Q+P) e_{x y} \\
& \Delta f_{y}=s+2 N e_{y y}
\end{aligned}
$$

Equations 1 and 4 may be transformed to a simpler set by introducing a scalar $\phi$, since the condition of incompressibility (6) implies

$$
\begin{aligned}
& u=-\frac{\partial \phi}{\partial y} \\
& v=\frac{\partial \phi}{\partial x} .
\end{aligned}
$$


With this expression, the equations reduce to a system with two unknowns,

$$
\begin{aligned}
& \frac{\partial s}{\partial x}-\frac{\partial}{\partial y}\left[\left(2 N-Q+\frac{P}{2}\right) \frac{\partial^{2} \phi}{\partial x^{2}}+\left(Q+\frac{P}{2}\right) \frac{\partial^{2} \phi}{\partial y^{2}}\right]=0 \\
& \frac{\partial s}{\partial y}+\frac{\partial}{\partial x}\left[\left(2 N-Q-\frac{P}{2}\right) \frac{\partial^{2} \phi}{\partial y^{2}}+\left(Q-\frac{P}{2}\right) \frac{\partial^{2} \phi}{\partial x^{2}}\right]=0
\end{aligned}
$$

and expression (7) become

$$
\begin{aligned}
\Delta f_{x} & =\left(Q+\frac{P}{2}\right)\left(\frac{\partial^{2} \phi}{\partial x^{2}}-\frac{\partial^{2} \phi}{\partial y^{2}}\right) \\
\Delta f_{y} & =s+2 N \frac{\partial^{2} \phi}{\partial x \partial y} .
\end{aligned}
$$

Eliminations of $s$ between the two equations (9) yields

$$
\left(Q-\frac{P}{2}\right) \frac{\partial^{4} \phi}{\partial x^{4}}+2(2 N-Q) \frac{\partial^{4} \phi}{\partial x^{2} \partial y^{2}}+\left(Q+\frac{P}{2}\right) \frac{\partial^{4} \phi}{\partial y^{4}}=0 .
$$

Solutions will be sought which are sinusoidal along $x \cdot \frac{\pi}{2}$ Hence, let

$$
\begin{aligned}
\phi l^{2} & =f(l y) \sin l x \\
s & =F(l y) \cos l x \\
u & =U(l y) \sin l x \\
v & =V(l y) \cos l x \\
\Delta f_{x} & =\tau(l y) \sin l x \\
\Delta f_{y} & =q(l y) \cos l x .
\end{aligned}
$$

Equation 11 becomes an ordinary differential equation. The function $f(\theta)$ satisfies the equation

$$
f^{\prime \prime \prime \prime}-2 m f^{\prime \prime}+k^{2} f=0 .
$$

The primes denote derivatives with respect to the argument $\theta=l y$. Let

$$
m=\frac{2 N-Q}{Q+\frac{P}{2}} \quad k^{2}=\frac{Q-\frac{P}{2}}{Q+\frac{P}{2}}
$$


In previous work and also in the discussion which follows the parameter

$$
\zeta=\frac{P}{2 Q}
$$

was considered. With this parameter, let

$$
\begin{aligned}
& m=\frac{2 N / Q-1}{1+\zeta} \\
& k^{2}=\frac{1-\zeta}{1+\zeta} .
\end{aligned}
$$

Substituting $\phi$ and $s$ in the first of Eqs. 9 determines $F$ in terms of $f$

$$
F=\left(2 N-Q+\frac{P}{2}\right) f^{\prime}-\left(Q+\frac{P}{2}\right) f^{\prime \prime \prime}
$$

All quantities are then expressed in terms of $f$, that is,

$$
\begin{aligned}
U l & =-f^{\prime} \\
V l & =f \\
\frac{\tau}{L} & =-f^{\prime \prime}-f \\
\frac{q}{L} & =(2 m+1) f^{\prime}-f^{\prime \prime \prime} .
\end{aligned}
$$

Let

$$
L=Q+\frac{P}{2}
$$

The physical significance of this coefficient is brought out by considering a deformation which is a uniform shear displacement

$$
\begin{aligned}
& u=\alpha y \\
& v=0 .
\end{aligned}
$$

The force on any plane perpendicular to the $y$ direction is given by Eq. 7. As a result,

$$
\begin{aligned}
& \Delta f_{x}=L \alpha \\
& \Delta f_{y}=0 .
\end{aligned}
$$

This represents a tangential shearing force $\Delta f_{x}$. The elastic coefficient $L$ represents a sliding rigidity on this particular plane under the existing state of initial stress. This has been referred to as the slide modulus. 
Another coefficient is $(\mathbf{8}, \mathbf{1 0})$

$$
\bar{M}=\bar{N}+\frac{P}{4}
$$

Using these coefficients,

$$
\begin{aligned}
& m=\frac{2 M-L}{L} \\
& k^{2}=\frac{L-P}{L} .
\end{aligned}
$$

For a medium which is isotropic in finite strain, $Q$ is given by $(10,13)$

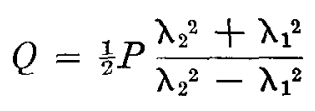

where $\lambda_{1}$ and $\lambda_{2}$ are the finite extension ratios in $x$ and $y$ directions in the state of initial stress. The slide modulus in this casc is

$$
L=P \frac{\lambda_{2}^{2}}{\lambda_{2}^{2}-\lambda_{1}^{2}}
$$

For a compression $P$ in the $x$ direction $\lambda_{1}<1$.

If, in addition to being isotropic in finite strain, the material is incompressible and obeys the finite stress-strain relations of an idealized rubber-type material,

$$
P=\mu_{0}\left(\lambda_{2}^{2}-\lambda_{1}^{2}\right)
$$

This material remains isotropic for plane incremental strain. The cocfficients $N$ and $Q$ become equal,

$$
N=Q=\frac{1}{2} \mu_{0}\left(\lambda_{1}^{2}+\lambda_{2}^{2}\right) .
$$

The coefficient $\mu_{0}$ is the shear modulus at vanishing initial stress $\left(\lambda_{1}=\lambda_{2}=1\right)$.

Attention is called to an important property of the parameters (17) and (18) for such a material. They become

$$
\begin{aligned}
\zeta & =\frac{\lambda_{2}{ }^{2}-\lambda_{1}{ }^{2}}{\lambda_{2}{ }^{2}+\lambda_{1}{ }^{2}} \\
m & =\frac{1}{1+\zeta}=\frac{\lambda_{1}{ }^{2}+\lambda_{1}{ }^{2}}{2 \lambda_{2}{ }^{2}} \\
k^{2} & =\frac{1-\zeta}{1+\zeta}=\frac{\lambda_{1}{ }^{2}}{\lambda_{2}{ }^{2}}
\end{aligned}
$$


Hence, they are independent of the rigidity coefficient $\mu_{0}$ and are completely determined by the magnitude of the finite initial strain.

In the case of a laminated medium composed of alternate layers of different properties, one type of material is defined by the elastic coefficients $L_{1}$ and $M_{1}$. The layers of this material are of equal thickness and under the compressive stress $P_{1}$. They occupy a fraction $\alpha_{1}$ of the total thickness. Layers of another material of coefficients $L_{2}, M_{2}$ are

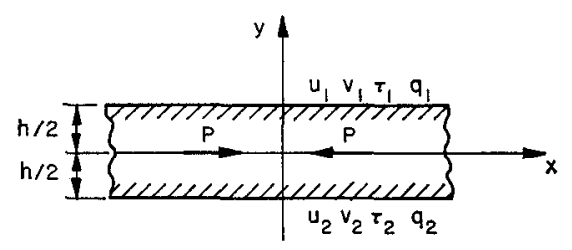

(a)

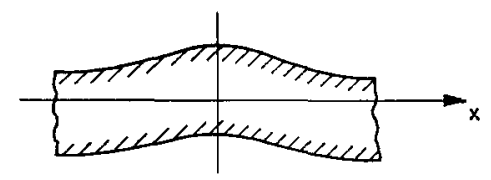

(b)

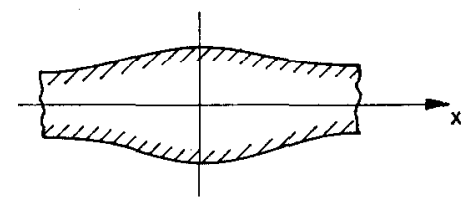

FIG. 2. Antisymmetric (a) and symmetric (b) deformation of an isolated layer with an initial compressive stress $P$.

sandwiched between the first and occupy a fraction $\alpha_{2}$ of the total thickness. They are also of equal thickness, and the compressive stress in them is $P_{2}$. Obviously,

$$
\alpha_{1}+\alpha_{2}=1
$$

and the total average initial stress in the composite medium is

$$
\alpha_{1} P_{1}+\alpha_{2} P_{2}=P \text {. }
$$

The properties of this medium may be approximated by considering an anisotropic continuum of coefficients (10)

$$
\begin{aligned}
& M=M_{1} \alpha_{1}+M_{2} \alpha_{2} \\
& L=\frac{1}{\frac{\alpha_{1}}{L_{1}}+\frac{\alpha_{2}}{L_{2}}}
\end{aligned}
$$


However, there are limitations to this approximation. It is valid only for deformations whose wave length is large enough relative to the thickness of the layers. Combining Eys. 23 and 30 and the value for $M$ from Eq. 32, the coefficient $N$ of the laminated medium can be derived as

$$
N=N_{1} \alpha_{1}+N_{2} \alpha_{2} .
$$

The coefficient $Q$ may also be derived from Eqs. 20 and 32 .

\section{ANALYSIS OF AN ISOLATED LAYER}

The next step is to consider an isolated layer of thickness $h$. The $x$ axis is located half way between the two faces (Fig. 2). Analysis of the most general deformation of this plate (which is sinusoidal along the $x$ direction) is most conveniently accomplished by superposition of two types of deformation, one antisymmetric with respect to the $x$ axis, as shown in Fig. 2a, and the other symmetric with respect to the same axis, as shown in Fig. $2 b$.

In the antisymmetric deformation the function $f(\theta)$ is an even function of the argument $\theta=l y$. The solution of the differential equation (15) which corresponds to this case is

$$
f=C_{1} \cosh \beta_{1} \theta+C_{2} \cosh \beta_{2} \theta .
$$

The constants of integration $C_{1}, C_{2}$ are determined by the boundary conditions. Let $\beta_{1}$ and $\beta_{2}$ denote the roots of the characteristic equation

$$
\beta^{4}-2 m \beta^{2}+k^{2}=0 .
$$

Hence,

$$
\begin{aligned}
& \beta_{1}=\sqrt{m+\sqrt{m^{2}-k^{2}}} \\
& \beta_{2}=\sqrt{m-\sqrt{m^{2}-\bar{k}^{2}}} .
\end{aligned}
$$

At this point something should be said about the choice of these roots, since there is a sign ambiguity involved in the definition of the square roots. The value of $\beta$ may be real, complex or imaginary. The following possible cases must be considered:

1. $m>0 m^{2}-k^{2}>0$. Two subcases must be distinguished:

(a) $k^{2}>0$. In this case both roots $\beta_{1}$ and $\beta_{2}$ are real, and their positive values may be chosen.

(b) $k^{2}<0$. Here the root $\beta_{1}$ is real, and the root $\beta_{2}$ is pure imaginary. Again, positive values may be chosen for $\beta_{1}$ and $\beta_{2} / i$.

2. $m<0 m^{2}-k^{2}>0$. In this case both roots are imaginary, and positive values are chosen for $\beta_{1} / i$ and $\beta_{2} / i$.

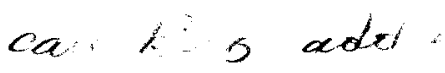


3. $m^{2}-k^{2}<0$, with $m$ assuming any real value, positive, negative or zero. In this case the roots are complex conjugates, and values for $\beta_{1}$ and $\beta_{2}$ are chosen with positive real parts.

The two limiting cases $m=0$ and $m^{2}-k^{2}=0$ must also be considered. The first does not give rise to any difficulty. The second case yields double roots and is discussed in detail in Section 4.

Two of these cases, $1(b)$ and 2, correspond to internal buckling (10). This can be recognized by putting $\beta^{2}=-\xi^{2}$ and writing the characteristic equation (35) in the form

$$
L \xi^{4}+2(2 M-L) \xi^{2}+L-P=0 .
$$

Internal buckling corresponds to the existence of one or two real roots $\xi$ for this equation (10). Therefore, internal buckling is represented by cases for which at least one $\operatorname{root} \beta$ is imaginary. If the parameters remain outside the range of internal buckling, the roots $\beta_{1}, \beta_{2}$ are either real or complex conjugate. In the latter case the solution (34) remains real if the constants of integration $C_{1}$ and $C_{2}$ are also chosen to be complex conjugates. In all other cases the solution (34) remains real with real values of the constants.

Designate by the subscript $a$ the values of $U, V, \tau$, and $q$ relative to the antisymmetric solution. They are functions of the argument $\theta$ and are designated as $U_{a}(\theta), V_{a}(\theta), \tau_{a}(\theta)$, and $q_{a}(\theta)$. Substituting solution (34) for $f$ into relations (19) determines the displacements

$$
\begin{aligned}
& l U_{a}(\theta)=-C_{1} \beta_{1} \sinh \beta_{1} \theta-C_{2} \beta_{2} \sinh \beta_{2} \theta \\
& l V_{a}(\theta)=C_{1} \cosh \beta_{1} \theta+C_{2} \cosh \beta_{2} \theta
\end{aligned}
$$

and the forces

$$
\begin{aligned}
& \frac{\tau_{a}(\theta)}{L}=-C_{1}\left(\beta_{1}^{2}+1\right) \cosh \beta_{1} \theta-C_{2}\left(\beta_{2}^{2}+1\right) \cosh \beta_{2} \theta \\
& \frac{q_{a}(\theta)}{L}=C_{1} \beta_{1}\left(\beta_{2}^{2}+1\right) \sinh \beta_{1} \theta+C_{2} \beta_{2}\left(\beta_{1}^{2}+1\right) \sinh \beta_{2} \theta
\end{aligned}
$$

In deriving these expressions use has been made of the relation

$$
\beta_{1}^{2}+\beta_{2}^{2}=2 m
$$

Next, write the values of these quantities at the upper face of the layer, that is, for

$$
\theta=\frac{l h}{2}=\gamma
$$


For simplicity, the variables at this point are designated by

$$
\begin{aligned}
U_{a} & =U_{a}(\gamma) \\
V_{a} & =V_{a}(\gamma) \\
\tau_{a} & =\tau_{a}(\gamma) \\
q_{a} & =q_{a}(\gamma) .
\end{aligned}
$$

Their expressions are given by the following equations,

$$
\begin{aligned}
U_{a} l & =-C_{1} \beta_{1} \sinh \beta_{1} \gamma-C_{2} \beta_{2} \sinh \beta_{2} \gamma \\
V_{a} l & =C_{1} \cosh \beta_{1} \gamma+C_{2} \cosh \beta_{2} \gamma \\
\frac{\tau_{a}}{L} & =-C_{1} \beta_{1}^{2} \cosh \beta_{1} \gamma-C_{2} \beta_{2}^{2} \cosh \beta_{2} \gamma-V_{a} l \\
\frac{q_{a}}{L} & =\beta_{1} \beta_{2}\left(C_{1} \beta_{2} \sinh \beta_{1} \gamma+C_{2} \beta_{1} \sinh \beta_{2} \gamma\right)-U_{a} l
\end{aligned}
$$

Solving the first two equations for $C_{1}$ and $C_{2}$ and substituting in the last two give

$$
\begin{aligned}
& C_{1}=-\frac{l}{\Delta_{a} \cosh \beta_{1} \gamma}\left(U_{a}+V_{a} \beta_{2} \tanh \beta_{2} \gamma\right) \\
& C_{2}=\frac{l}{\Delta_{a} \cosh \beta_{2} \gamma}\left(U_{a}+V_{a} \beta_{1} \tanh \beta_{1} \gamma\right)
\end{aligned}
$$

and

$$
\begin{aligned}
& \frac{\tau_{a}}{l L}=a_{11} U_{a}+a_{12} V_{a} \\
& \frac{q_{a}}{l L}=a_{12} U_{a}+a_{22} V_{a} .
\end{aligned}
$$

Let

$$
\begin{aligned}
& a_{11}=\frac{\beta_{1}^{2}-\beta_{2}^{2}}{\Delta_{a}} \\
& a_{12}=\beta_{1} \beta_{2} \frac{\Delta_{s}}{\Delta_{a}}-1 \\
& a_{22}=\beta_{1} \beta_{2} \frac{\left(\beta_{1}^{2}-\beta_{2}^{2}\right)}{\Delta_{a}} \tanh \beta_{1} \gamma \tanh \beta_{2} \gamma
\end{aligned}
$$

and

$$
\begin{aligned}
& \Delta_{s}=\beta_{1} \tanh \beta_{2} \gamma-\beta_{2} \tanh \beta_{1} \gamma \\
& \Delta_{a}=\beta_{1} \tanh \beta_{1} \gamma-\beta_{2} \tanh \beta_{2} \gamma
\end{aligned}
$$


It is also convenient to write these expressions in a more compact form by letting

$$
\begin{aligned}
& x_{1}=\beta_{1} \tanh \beta_{1} \gamma \\
& x_{2}=\beta_{2} \tanh \beta_{2} \gamma
\end{aligned}
$$

This gives

$$
\begin{aligned}
& a_{11}=\frac{\beta_{1}^{2}-\beta_{2}^{2}}{x_{1}-x_{2}} \\
& a_{12}=\frac{\left(\beta_{1}^{2}+1\right) x_{2}-\left(\beta_{2}^{2}+1\right) x_{1}}{x_{1}-x_{2}} \\
& a_{22}=\frac{\left(\beta_{1}^{2}-\beta_{2}^{2}\right) x_{1} x_{2}}{x_{1}-x_{2}}=a_{11} x_{1} x_{2} .
\end{aligned}
$$

The displacements and stresses on the bottom side of the layer, obtained by substituting $\theta=-\gamma$ into relations (38) and (39), are

$$
\begin{aligned}
& U_{a}(-\gamma)=-U_{a} \\
& V_{a}(-\gamma)=V_{a} \\
& \tau_{a}(-\gamma)=\tau_{a} \\
& q_{a}(-\gamma)=-q_{a} .
\end{aligned}
$$

By a similar procedure the solution is obtained for the symmetric deformation illustrated in Fig. 2b. In this case the function $f(\theta)$ is odd, that is,

$$
f(\theta)=C_{1} \sinh \beta_{1} \theta+C_{2} \sinh \beta_{2} \theta .
$$

The variables for this case are designated by the subscript $s$. They are $U_{s}(\theta), V_{s}(\theta), \tau_{s}(\theta), q_{s}(\theta)$. Their values at the upper face of the layer are denoted by

$$
\begin{aligned}
U_{s}(\gamma) & =U_{s} \\
V_{s}(\gamma) & =V_{s} \\
\tau_{s}(\gamma) & =\tau_{s} \\
q_{s}(\gamma) & =q_{s} .
\end{aligned}
$$

For this case we derive

$$
\begin{aligned}
& \frac{\tau_{s}}{l L}=b_{11} U_{s}+b_{12} V_{s} \\
& \frac{q_{s}}{l L}=b_{12} U_{s}+b_{22} V_{s}
\end{aligned}
$$


with

$$
\begin{aligned}
& b_{11}=\frac{\beta_{1}{ }^{2}-\beta_{2}^{2}}{\Delta_{s}} \tanh \beta_{1} \gamma \tanh \beta_{2} \gamma \\
& b_{12}=\beta_{1} \beta_{2} \frac{\Delta_{a}}{\Delta_{s}}-1 \\
& b_{22}=\beta_{1} \beta_{2} \frac{\beta_{1}^{2}-\beta_{2}^{2}}{\Delta_{s}} .
\end{aligned}
$$

Values of the variables on the bottom side of the layer are

$$
\begin{aligned}
& U_{s}(-\gamma)=U_{s} \\
& V_{s}(-\gamma)=-V_{s} \\
& \tau_{s}(-\gamma)=-\tau_{s} \\
& q_{s}(-\gamma)=q_{s} .
\end{aligned}
$$

Next, superpose the two solutions obtained above. Denote by $U_{1}, V_{1}$, $\tau_{1}, q_{1}$, the values of the variables at the top of the layer and by $U_{2}, V_{2}$, $\tau_{2}, q_{2}$ the values of the same variables on the bottom side (Fig. 2).

For the top side, write

$$
\begin{aligned}
U_{1} & =U_{a}+U_{s} & V_{1} & =V_{a}+V_{s} \\
\tau_{1} & =\tau_{a}+\tau_{s} & q_{1} & =q_{a}+q_{s},
\end{aligned}
$$

and for the bottom side,

$$
\begin{aligned}
U_{2} & =-U_{a}+U_{s} & V_{2} & =V_{a}-V_{s} \\
\tau_{2} & =\tau_{a}-\tau_{s} & q_{2} & =-q_{a}+q_{s} .
\end{aligned}
$$

Then,

$$
\begin{array}{ll}
U_{a}=\frac{1}{2}\left(U_{1}-U_{2}\right) & U_{s}=\frac{1}{2}\left(U_{1}+U_{2}\right) \\
V_{a}=\frac{1}{2}\left(V_{1}+V_{2}\right) & V_{s}=\frac{1}{2}\left(V_{1}-V_{2}\right) .
\end{array}
$$

Substituting these values in expressions (45) and (53), the values of $\tau_{a}, q_{a}, \tau_{s}, q_{s}$ are obtained in terms of the top and bottom displacements. Finally, introducing these values into Eqs. 56 and 57 gives

$$
\left.\left[\begin{array}{l}
\tau_{1} \\
q_{1} \\
\tau_{2} \\
q_{2}
\end{array}\right]=l L\left(\begin{array}{rrrr}
A_{1} & A_{2} & -A_{4} & A_{5} \\
A_{2} & A_{3} & -A_{5} & A_{6} \\
A_{4} & A_{5} & -A_{1} & A_{2} \\
-A_{5} & -A_{6} & A_{2} & -A_{3}
\end{array}\right]\right)\left(\begin{array}{c}
U_{1} \\
V_{1} \\
U_{2} \\
V_{2}
\end{array}\right] .
$$


There are six distinct coefficients in this matrix expressed in terms of the six coefficients (49) and (54). They are

$$
\begin{array}{ll}
A_{1}=\frac{1}{2}\left(a_{11}+b_{11}\right) & A_{4}=\frac{1}{2}\left(a_{11}-b_{11}\right) \\
A_{2}=\frac{1}{2}\left(a_{12}+b_{12}\right) & A_{5}=\frac{1}{2}\left(a_{12}-b_{12}\right) \\
A_{3}=\frac{1}{2}\left(a_{22}+b_{22}\right) & A_{6}=\frac{1}{2}\left(a_{22}-b_{22}\right) .
\end{array}
$$

A more convenient way to write Eq. 59 is to introduce an invariant which represents a quantity proportional to the potential energy. Let

$I=a_{11} U_{a}^{2}+2 a_{12} U_{a} V_{a}+a_{12} V_{a}^{2}+b_{11} U_{s}^{2}+2 b_{12} U_{s} V_{s}+b_{22} V_{s}^{2}$.

Substituting Eqs. 58 into Eq. 61 gives

$$
\begin{aligned}
I=\frac{1}{2} A_{1}\left(U_{1}^{2}+U_{2}^{2}\right) & -A_{1} U_{1} U_{2}+\frac{1}{2} A_{3}\left(V_{1}^{2}+V_{2}^{2}\right)+A_{6} V_{1} V_{2} \\
& +A_{2}\left(U_{1} V_{1}-U_{2} V_{2}\right)+A_{5}\left(U_{1} V_{2}-U_{2} V_{1}\right) .
\end{aligned}
$$

Equations 59 are then written

$$
\begin{aligned}
\tau_{1} & =l L \frac{\partial I}{\partial U_{1}} & \tau_{2} & =-l L \frac{\partial I}{\partial U_{2}} \\
q_{1} & =l L \frac{\partial I}{\partial V_{1}} & q_{2} & =-l L \frac{\partial I}{\partial V_{2}} .
\end{aligned}
$$

4. SOME LIMITING CASES

In this section some particular cases of special interest will be examined.

An obvious simplification should result by considering the layer thickness to be infinite. This should yield the solution for the half space. (11) In order to retain its physical significance, the solution must be such that internal buckling is excluded. This corresponds to cases $1(a)$ and 3 discussed in Section 3 , and the roots $\beta_{1}$ and $\beta_{2}$ are real and positive or complex conjugate with positive real parts.

Since

$$
\gamma=\frac{1}{2} l h
$$

assuming an infinite thickness amounts to setting

$$
\gamma \doteq \infty \text {. }
$$

The same limiting case is also obtained for a finite thickness when

$$
l=\infty,
$$

that is, when the wave length becomes very small. Either case results, 
of course, from the geometric interpretation of the parameter $\gamma$ as proportional to the ratio of the thickness to the wave length.

Taking into account the positive signs of the real parts in the values of $\beta_{1}$ and $\beta_{2}$,

$$
\begin{aligned}
& \lim _{\gamma \rightarrow \infty} \tanh \beta_{1} \gamma=1 \\
& \lim _{\gamma \rightarrow \infty} \tanh \beta_{2} \gamma=1 .
\end{aligned}
$$

Hence, the limiting values of the coefficients (Eqs. 49 and 54) are

$$
\begin{aligned}
& a_{11}=b_{11}=\beta_{1}+\beta_{2} \\
& a_{12}=b_{12}=\beta_{1} \beta_{2}-1 \\
& a_{22}=b_{22}=\beta_{1} \beta_{2}\left(\beta_{1}+\beta_{2}\right) .
\end{aligned}
$$

The coefficients (Eqs. 60) become

$$
\begin{aligned}
& A_{1}=a_{11} \\
& A_{2}=a_{12} \\
& A_{3}=a_{22}
\end{aligned}
$$

and

$$
A_{4}=A_{5}=A_{6}=0 .
$$

Reference to Eq. 59 shows that the top and bottom sides of the plate are now decoupled.

The roots $\beta_{1}$ and $\beta_{2}$ do not have to be evaluated separately. Because a sign has been chosen for the roots, it is possible to write without ambiguity

$$
\begin{aligned}
& \beta_{1} \beta_{2}=k \\
& \beta_{1}+\beta_{2}=\sqrt{2(m+k)} .
\end{aligned}
$$

The relation between surface forces and displacement at the top of the infinitely thick layer becomes

$$
\begin{aligned}
& \frac{\tau}{l L}=a_{11} U+a_{12} V \\
& \frac{q}{l L}=a_{12} U+a_{22} V,
\end{aligned}
$$

with the values of Eqs. 68 for the coefficients. This result coincides with one obtained earlier by the author (11) for the lower half space.

Similar expressions are obtained for the upper half space simply by 
reversing the sign of $a_{11}$ and $a_{22}$. This is readily seen by using the last two equations of the matrix equation (59).

It is convenient to express Eqs. 72 for the lower half space by using the invariant

$$
I_{l}=\frac{1}{2} a_{11} U^{2}+a_{12} U V+\frac{1}{2} a_{22} V^{2} .
$$

In this expression the coefficients $a_{i j}$ are given by Eqs. 68 and 71 . They depend only on the elastic coefficients and the initial stress in the medium, and are independent of the wave length. Equations 72 may then be written

$$
\begin{aligned}
& \tau=l L \frac{\partial I_{l}}{\partial U} \\
& q=l L \frac{\partial I_{l}}{\partial V} .
\end{aligned}
$$

Similarly for the upper half space, the invariant,

$$
I_{u}=\frac{1}{2} a_{11} U^{2}-a_{12} U V+\frac{1}{2} a_{12} V^{2}
$$

is introduced. Relations between surface forces and displacements for the upper half space are written as

$$
\begin{gathered}
\tau=-l L \frac{\partial I_{u}}{\partial U} \\
q=-l L \frac{\partial I_{u}}{\partial V} .
\end{gathered}
$$

Another limiting case is obtained when the characteristic equation (35) has a double root. This is obtained when

$$
m^{2}-k^{2}=0 \text {. }
$$

The double root is

$$
\beta_{1}=\beta_{2}=\sqrt{m}=\beta
$$

Exclusion of internal buckling in this case requires that $m$ be positive. When $\beta_{1}=\beta_{2}$, the coefficients $a_{i j}$ and $b_{i j}$ in Eqs. 46 and 54 become undetermined, and their true limiting value must be found. To do this, let

$$
\begin{aligned}
& \beta_{1}=\beta+\epsilon \\
& \beta_{2}=\beta-\epsilon
\end{aligned}
$$

and expand the hyperbolic functions of expressions (46) and (54) to the first order in $\epsilon$. After cancellations of common factors in numerator and denominator, the limiting values are derived by putting $\epsilon=0$. 
For the antisymmetric case,

$$
\begin{aligned}
a_{11} & =\frac{4 \beta \cosh ^{2} \beta \gamma}{\sinh 2 \beta \gamma+2 \beta \gamma} \\
a_{12} & =\frac{\beta^{2} \sinh 2 \beta \gamma-2 \beta \gamma}{\sinh 2 \beta \gamma+2 \beta \gamma}-1 \\
a_{22} & =\frac{4 \beta^{3} \sinh ^{2} \beta \gamma}{\sinh 2 \beta \gamma+2 \beta \gamma} .
\end{aligned}
$$

In the symmetric case the limiting values are

$$
\begin{aligned}
b_{11} & =\frac{4 \beta \sinh ^{2} \beta \gamma}{\sinh 2 \beta \gamma-2 \beta \gamma} \\
b_{12} & =\frac{\beta^{2} \sinh 2 \beta \gamma+2 \beta \gamma}{\sinh 2 \beta \gamma-2 \beta \gamma}-1 \\
b_{22} & =\frac{4 \beta^{3} \cosh ^{2} \beta \gamma}{\sinh 2 \beta \gamma-2 \beta \gamma} .
\end{aligned}
$$

Condition 77 for the existence of double roots may be written

$$
4 N(N-Q)+\frac{P^{2}}{4}=0 .
$$

It is interesting to note that it cannot occur for $N>Q$. The particular case

$$
N=Q \quad P=0
$$

represents the classical case of an isotropic medium free of initial stress. The characteristic roots in this case degenerate into the values

$$
\beta_{1}=\beta_{2}=\beta=1 \text {. }
$$

Substituting $\beta=1$ into Eqs. 80 and 81 , the coefficients for the antisymmetric case become

$$
\begin{aligned}
a_{11} & =\frac{4 \cosh ^{2} \gamma}{\sinh 2 \gamma+2 \gamma} \\
a_{12} & =-\frac{4 \gamma}{\sinh 2 \gamma+2 \gamma} \\
a_{22} & =\frac{4 \sinh ^{2} \gamma}{\sinh 2 \gamma+2 \gamma}
\end{aligned}
$$


and for the symmetric case

$$
\begin{aligned}
& b_{11}=\frac{4 \sinh ^{2} \gamma}{\sinh 2 \gamma-2 \gamma} \\
& b_{12}=\frac{4 \gamma}{\sinh 2 \gamma-2 \gamma} \\
& b_{22}=\frac{4 \cosh ^{2} \gamma}{\sinh 2 \gamma-2 \gamma}
\end{aligned}
$$

These coefficients are identical with those obtained by solving directly the classical problem of the isotropic and incompressible elastic plate without initial stress. The basic equations for this classical case are, of course, derived immediately by putting $N=Q$ and $P=0$ into Eqs. 9, 10, and 11. It is seen that the latter degenerates into the wellknown biharmonic equation.

\section{GENERAL EQUATIONS FOR THE STABILITY OF MULTILAYERED SYSTEMS}

The previous results make it possible to obtain very simply and in a systematic way the stability equations for a system of superposed layers under initial stress. In the preceding section it was assumed that the state of initial stress in the $x, y$ plane is reduced to a single principal stress component $S_{11}=-P$ acting in a direction parallel with the layer. In order to deal with more general problems, such as the case of layers embedded in an infinite medium, the case where the initial stress includes a principal component $S_{22}$ acting perpendicularly to the layer (Fig. 3) must be examined.

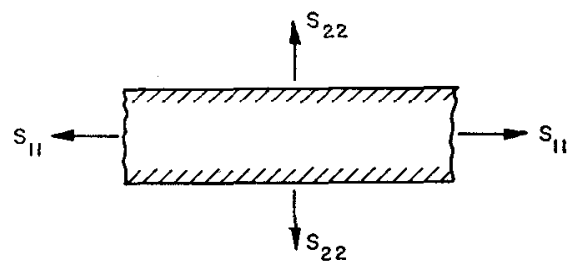

Frg. 3. State of initial stress in a layer when an initial component $S_{22}$ is present.

As will now be shown, all expressions obtained in the previous section are valid for this case, provided $P$ is defined as

$$
P=S_{22}-S_{11} \text {. }
$$

With this definition for $P$, the equilibrium equations (1) are obviously valid as can be seen by applying the more general equations (A1) of the Appendix. 
Equations 63 also remain valid when $P$ is given the value in Eq. 87. However, the physical significance of $\tau$ and $q$ must be given a new interpretation. This can be seen as follows.

The incremental forces given by Eqs. A2 in the Appendix are

$$
\begin{aligned}
& \Delta f_{x}=s_{12}-S_{22} \omega-S_{11} e_{x y} \\
& \Delta f_{y}=s_{22}+S_{22} e_{x x} .
\end{aligned}
$$

This may be written

$$
\begin{aligned}
& \Delta f_{x}=s_{12}+P e_{x y}-S_{22} \frac{\partial v}{\partial x} \\
& \Delta f_{y}=s_{22}+S_{22} \frac{\partial u}{\partial x}
\end{aligned}
$$

where $P=S_{22}-S_{11}$.

The terms

$$
\begin{aligned}
& \Delta^{\prime} f_{x}=s_{12}+P e_{x y}=(2 Q+P) e_{x y} \\
& \Delta^{\prime} f_{y}=s_{22}=s+2 N e_{y y}
\end{aligned}
$$

coincide with expressions (7), except that $P$ is now the difference (Eq. 87) of the principal stresses. Equations 89 show that $\Delta^{\prime} f_{x}$ and $\Delta^{\prime} f_{y}$ are the incremental stress components along directions which are tangent and normal to the deformed surface. Note that these stresses are now referred to unit areas after deformation.

It is concluded that Eqs. 45, 53 and 63 are valid in the more general case where $S_{22} \neq 0$, provided $\tau$ and $q$ are interpreted as represcnting

$$
\begin{aligned}
& \Delta^{\prime} f_{x}=\tau \sin l x \\
& \Delta^{\prime} f_{y}=q \cos l x .
\end{aligned}
$$

Consider now a system of superposed layers with perfect adherence along parallel intcrfaces and cmbedded between two scmi-infinite media

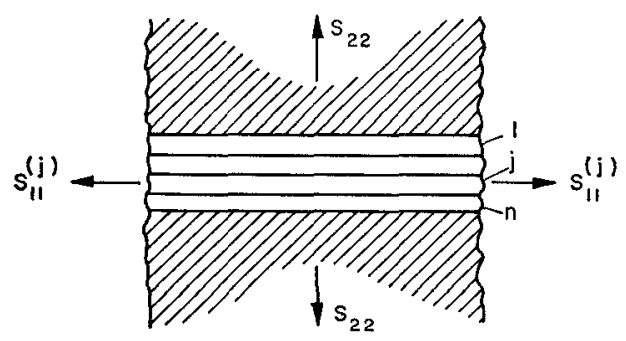

FIG. 4. Superposed layers embedded between two semi-infinite media. 
(Fig. 4). The $y$ axis is oriented normally to the layers. There are $n$ layers numbered from 1 to $n$ starting at the top.

The state of initial stress is represented by a normal component $S_{22}$ the same for all layers. The $x$ component $S_{11}$ of the initial stress may be different in each layer. Designate by $S_{11}^{(j)}$ its value in the $j^{\text {th }}$ layer. In this layer the stress difference is

$$
P_{j}=S_{22}-S_{11}^{(j)} .
$$

The displacements of the upper face of the $j^{\text {th }}$ layer are denoted by $U_{j}, V_{j}$ and that of the lower face by $U_{j+1}, V_{j+1}$.

The properties of the $j^{\text {th }}$ layer are completely defined by the invariant $(62)$. Its value for the $j^{\text {th }}$ layer is written

$$
\begin{aligned}
I_{j}=\frac{1}{2} A_{1}{ }^{j}\left(U_{j}^{2}+\right. & \left.U_{j+1^{2}}\right)-A_{4}{ }^{j} U_{j} U_{j+1}+\frac{1}{2} A_{3^{j}}\left(V_{j}{ }^{2}+V_{j+1}{ }^{2}\right) \\
& +A_{6}{ }^{j} V_{j} V_{j+1}+A_{2}{ }^{j}\left(U_{j} V_{j}-U_{j+1} V_{j+1}\right) \\
& +A_{5}{ }^{j}\left(U_{j} V_{j+1}-U_{j+1} V_{j}\right) .
\end{aligned}
$$

The coefficients $A_{1}{ }^{j}, A_{2}{ }^{j}$ etc., are the values of $A_{1}, A_{2}$ etc., for the $j^{\text {th }}$ layer as defined by Eqs. 60 . They are functions of the following parameters :

(a) The two elastic coefficients $N_{j}$ and $Q_{j}$ of the $j^{\text {th }}$ layer.

(b) The stress difference $P_{j}=S_{22}-S_{11}^{(j)}$ of the $j^{\text {th }}$ layer.

(c) The variable $\gamma_{j}=\frac{1}{2} l h_{j}$ where $h_{j}$ is the thickness of the $j^{\text {th }}$ layer.

Denote by

$$
L_{j}=Q_{j}+\frac{1}{2} P_{j}
$$

the slide modulus of the same layer. The stresses at the bottom of the layer are given by Eqs. 63,

$$
\begin{aligned}
\tau_{j+1}{ }^{\prime} & =-l L_{j} \frac{\partial I_{j}}{\partial U_{j+1}} \\
q_{j+1}{ }^{\prime} & =-l L_{j} \frac{\partial I_{j}}{\partial V_{j+1}} .
\end{aligned}
$$

The stresses at the top of the $(j+1)^{\text {th }}$ layer are given by the same Eqs. 63,

$$
\begin{aligned}
\tau_{j+1} & =l L_{j+1} \frac{\partial I_{j+1}}{\partial U_{j+1}} \\
q_{j+1} & =l L_{j+1} \frac{\partial I_{j+1}}{\partial V_{j+1}}
\end{aligned}
$$


Since the stresses $\Delta^{\prime} f_{x}$ and $\Delta^{\prime} f_{y}$ given by Eq. 91 must be continuous at an interface, expressions (95) and (96) must be equal.

This yields

$$
\begin{aligned}
& \frac{\partial}{\partial U_{j+1}}\left(L_{j} I_{j}+L_{j+1} I_{j+1}\right)=0 \\
& \frac{\partial}{\partial V_{j+1}}\left(L_{j} I_{j}+L_{j+1} I_{j+1}\right)=0 .
\end{aligned}
$$

These two recurrence equations relate six variables, namely the two displacement components $U_{j}$ and $V_{j}$ at three successive interfaces.

At the top and bottom interfaces the equations take a special form because of the infinite thickness of the upper and lower media. It is necessary to introduce the previously defined invariants $I_{l}$ and $I_{u}$ for the lower and upper half spaces as given by Eqs. 73 and 75 . In the present case they are written

$$
\begin{aligned}
& I_{l}=\frac{1}{2} a_{11} U_{n+1^{2}}+a_{12} U_{n+1} V_{n+1}+\frac{1}{2} a_{22} V_{n+1}{ }^{2} \\
& I_{u}=\frac{1}{2} a_{11}{ }^{\prime} U_{1}{ }^{2}-a_{12}{ }^{\prime} U_{1} V_{1}+\frac{1}{2} a_{22}{ }^{\prime} V_{1}{ }^{2}
\end{aligned}
$$

As for the other interfaces, the continuity of the stress $\Delta^{\prime} f_{x}$ and $\Delta^{\prime} f_{y}$ at the top and bottom interfaces can be expressed, by four additional equations :

$$
\begin{array}{ll}
\frac{\partial}{\partial U_{1}}\left(L_{1} I_{1}+L^{\prime} I_{u}\right)=0 & \frac{\partial}{\partial V_{1}}\left(L_{1} I_{1}+L^{\prime} I_{u}\right)=0 \\
\frac{\partial}{\partial U_{n+1}}\left(L_{n} I_{n}+L I_{l}\right)=0 & \frac{\partial}{\partial V_{n+1}}\left(L_{n} I_{n}+L I_{l}\right)=0 .
\end{array}
$$

In Eqs. 99, $L^{\prime}$ denotes the slide modulus of the upper half space and $L$ the slide modulus of the lower half space.

Equations 97 and 99 constitute a system of $2(n+1)$ homogeneous
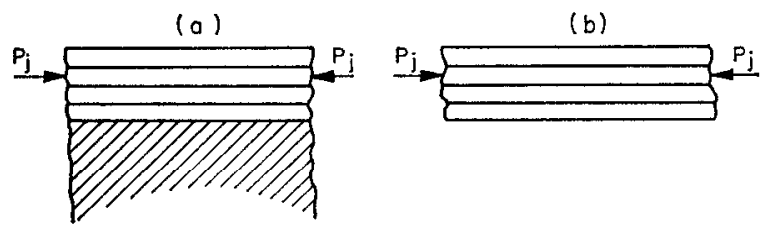

FIG. 5. (a) Superposed layers resting on a half space and free at the top.

(b) Superposed layers free at top and bottom.

equations for the $2(n+1)$ displacement variables $U_{j}$ and $V_{j}$. The characteristic stability equation is obtained by equating the determinant of this system to zero. 
For layers resting on a semi-infinite half space and free at the top surface (Fig. 5a'), $L^{\prime}=0$. Equations 99 are then replaced by

$$
\begin{array}{ll}
\frac{\partial I_{1}}{\partial U_{1}}=0 & \frac{\partial I_{1}}{\partial V_{1}}=0 \\
\frac{\partial}{\partial U_{n+1}}\left(L_{n} I_{n}+L I_{l}\right)=0 & \frac{\partial}{\partial V_{n+1}}\left(L_{n} I_{n}+L I_{l}\right)=0 .
\end{array}
$$

If the top and bottom surfaces of the layers are both free (Fig. 5b), then $L^{\prime}=L=0$ and Eqs. 99 become

$$
\begin{array}{ll}
\frac{\partial I_{1}}{\partial U_{1}}=0 & \frac{\partial I_{1}}{\partial V_{1}}=0 \\
\frac{\partial I_{n}}{\partial U_{n+1}}=0 & \frac{\partial I_{n}}{\partial V_{n+1}}=0 .
\end{array}
$$

A further simplification of the general formalism is obtained by introducing a single invariant which corresponds to the potential energy of the complete system. Let

$$
\mathfrak{J}=L I_{l}+\sum^{j} L_{j} I_{j}+L^{\prime} I_{u} .
$$

The stability equations become

$$
\frac{\partial \mathfrak{d}}{\partial U_{j}}=0 \quad \frac{\partial \mathscr{d}}{\partial V_{j}}=0 .
$$

These $2(n+1)$ equations include all three cases of free and embedded layers formulated by Eqs. 99, 100, and 101.

It is of interest to examine the case where the various materials obey the finite stress-strain relations (Eq. 27) of rubber-type elasticity. Here, a system originally stress-free is brought to an initial state of finite homogeneous strain represented by the extension ratio $\lambda_{1}$ and $\lambda_{2}$ in the $x, y$ plane. As shown by expressions (29), the parameters $m$, $k^{2}$ and $\zeta$ are then identical for all materials. They may be expressed in terms of a single variable, say $\lambda_{1}{ }^{2} / \lambda_{2}{ }^{2}$ or $\zeta$. The only other parameter in the values of $I_{j}$ is $\gamma$, which for the $j^{\text {th }}$ layer of thickness $h_{j}$ is written

$$
\gamma_{j}=\frac{1}{2} l h_{j}
$$

If the thickness of one of the layers is chosen as reference, say $h_{1}$, then

$$
\gamma_{j}=\left(h_{j} / h_{1}\right) \gamma
$$


where $\gamma=\frac{1}{2} h_{1} l$. Hence, the values of $I_{j}$ contain the variable $\gamma$ and the $(n-1)$ thickness ratios as parameters. Finally, taking into account Eqs. 26 and $27, L_{j}$ may be written as

$$
L_{j}=\mu_{0 j} \lambda_{2}{ }^{2}
$$

where $\mu_{0 j}$ is the stress-free modulus of the $j^{\text {th }}$ layer. Hence, in all Eqs. 103, the common factor $\lambda_{2}{ }^{2}$ cancels out. This brings out the ratios of the moduli $\mu_{0 j}$ as another set of parameters. When these rigidity ratios are given along with the various thickness ratios, the characteristic equation becomes a relation between the two variables $\zeta$ and $\gamma$. The first characterizes the initial stress and the second the wave length. The minimum value of $\zeta$ as a function of $\gamma$ yields the buckling stress and wave length.

The preceding analysis has assumed perfect adherence. If the layers are allowed to slip without friction at the interface, the following condition must be satisfied,

$$
\tau=0,
$$

since the tangential stresses must remain zero. The displacements $U_{j}$ in this case are not continuous. However, they may be eliminated from the equations by using condition (107) for perfect slip. This leaves a system of equations containing only the normal displacement $V_{j}$ at the slipping interface. This normal component is continuous.

A remark must be made here regarding the continuity of $q$ when such slippage occurs. Since this value is evaluated at the displaced points and since these points do not coincide due to slippage, the values of $q$ are not exactly continuous. However, the difference is a second order quantity, and in a first order theory interfacial values of $q$ may be assumed equal to each layer. The case of perfect slippage is illustrated by an example in a forthcoming paper in this Journal.

The recurrence equations derived above contain relatively simple coefficients and constitute a system of linear equations containing not more than six variables at a time.

It is possible to formulate the stability problem by a different procedure which may have advantages for numerical work with digital computers. The procedure was developed by Haskell for the formally similar problem of wave propagation in multilayered media (14). Equations 59 may be written in the form

$$
\left[\begin{array}{l}
\tau_{1} \\
q_{1} \\
l U_{1} \\
l V_{1}
\end{array}\right)=\Re\left(\begin{array}{l}
\tau_{2} \\
q_{2} \\
l U_{2} \\
l V_{2}
\end{array}\right] .
$$


The matrix is

$$
\Re=\left(\begin{array}{cccc}
B_{1} & B_{2} & L B_{5} & L B_{6} \\
B_{3} & B_{4} & -L B_{6} & L B_{7} \\
\frac{1}{L} B_{8} & \frac{1}{L} B_{9} & B_{1} & -B_{3} \\
-\frac{1}{L} B_{9} & \frac{1}{L} B_{10} & -B_{2} & B_{4}
\end{array}\right) .
$$

The elements $B_{k}$ are functions only of $a_{i j}$ and $b_{i j}$. With

$$
\Delta=\left(a_{12}-b_{12}\right)^{2}-\left(a_{11}-b_{11}\right)\left(a_{22}-b_{22}\right)
$$

they are

$$
\begin{aligned}
& B_{1}=\frac{1}{\Delta}\left[\left(a_{12}^{2}-b_{12}^{2}\right)-\left(a_{11}+b_{11}\right)\left(a_{22}-b_{22}\right)\right] \\
& B_{2}=\frac{2}{\Delta}\left(a_{11} b_{12}-a_{12} b_{11}\right) \\
& B_{3}=\frac{2}{\Delta}\left(a_{12} b_{22}-a_{22} b_{12}\right) \\
& B_{4}=\frac{1}{\Delta}\left[\left(a_{22}+b_{22}\right)\left(a_{11}-b_{11}\right)-\left(a_{12}^{2}-b_{12}^{2}\right)\right] \\
& B_{5}=\frac{2}{\Delta}\left[a_{12}^{2} b_{11}-a_{11} b_{12}^{2}-a_{11} b_{11}\left(a_{22}-b_{22}\right)\right] \\
& B_{6}=\frac{2}{\Delta}\left[-a_{12} b_{12}\left(a_{12}-b_{12}\right)+a_{11} a_{22} b_{12}-a_{12} b_{11} b_{22}\right] \\
& B_{7}=\frac{2}{\Delta}\left[a_{22} b_{22}\left(a_{11}-b_{11}\right)+a_{22} b_{12}^{2}-a_{12}^{2} b_{22}\right] \\
& B_{8}=-\frac{2}{\Delta}\left(a_{22}-b_{22}\right) \\
& B_{9}=-\frac{2}{\Delta}\left(a_{12}-b_{12}\right) \\
& B_{10}=\frac{2}{\Delta}\left(a_{11}-b_{11}\right) .
\end{aligned}
$$

The matrix equation (108) relates the values of the variables at two successive interfaces. Obviously the values at the top and bottom interfaces satisfy the equations 


$$
\left(\begin{array}{c}
\tau_{1} \\
q_{1} \\
l U_{1} \\
l V_{1}
\end{array}\right)=\prod_{j=1}^{n} \Re_{j}\left(\begin{array}{c}
\tau_{n+1} \\
q_{n+1} \\
l U_{n+1} \\
l V_{n+1}
\end{array}\right)
$$

The product $\prod_{j=1}^{n} \mathfrak{M}_{j}$ of the $\mathfrak{M}_{j}$ matrices for each layer is a $4 \times 4$ matrix. If the top and bottom faces are free, let $\tau_{1}=q_{1}=\tau_{n+1}$ $=q_{n+1}=0$, and Eq. 112 reduces to two homogencous equations for the two unknowns $U_{n+1}$ and $V_{n+1}$. If the layers lie over a half space, $\tau_{n+1}$ and $q_{n+1}$ are replaced by their values in terms of $U_{n+1}$ and $V_{n+1}$ for the half space and are again led to a $2 \times 2$ system of equations. If the layers are embedded between two half spaces, the values of $\tau_{1}, q_{1}$, $\tau_{n+1}, q_{n+1}$ are expressed in terms of $U_{1}, V_{1}$ and $U_{n+1}, V_{n+1}$. This case results in a system of four equations.

In using this procedure attention must be called to the case where the wave length is sufficiently small so that the top and bottom faces of a layer become decoupled. This happens when

$$
a_{i j} \approx b_{i j}
$$

In this case the layer may be replaced by a half space.

The procedure and formulas proposed here for the mechanics of layered media are quite general and are not restricted to incompressible media or stability problems. The matrix coefficients (Eqs. 111) are given a new form which is immediately applicable to a large category of problems, including wave propagation in anisotropic multilayered media. The recurrence equations (103) or the matrix multiplication process of Eqs. 112 provide a systematic way of programming the numerical work for the solution of problems in the mechanics of layered media when a large number of layers are involved and the use of a large capacity digital computer is available.

A variational principle is readily obtained from Eqs. 103, that is,

$$
\delta \mathfrak{d}=0 .
$$

In this principle the interfacial displacement $U_{i}$ and $V_{i}$ are given arbitrary variations. Equation 114 is also a consequence of the physical interpretation of $d$ as the total incremental energy of the multilayered system expressed in terms of the interfacial displacements.

\section{APPENDIX}

A brief outline is given here of previous results. The general equations were derived earlier $(1,2,3,9)$. For the case of plane strain and constant body force they were briefly rederived in a more recent paper (4). The results for this case are repeated here. The initial stresses are denoted by $S_{11}, S_{22}, S_{12}$. The incremental stresses referred to axes rotated by an angle $\omega$ are denoted by $s_{11}, s_{22}, s_{12}$. They satisfy the equilibrium conditions 
$\frac{\partial s_{11}}{\partial x}+\frac{\partial s_{12}}{\partial y}+\rho Y \omega-2 S_{12} \frac{\partial \omega}{\partial x}+\left(S_{11}-S_{22}\right) \frac{\partial \omega}{\partial y}$

$$
+\frac{\partial S_{11}}{\partial x} e_{y y}-\left(\frac{\partial S_{11}}{\partial y}+\frac{\partial S_{12}}{\partial x}\right) e_{x y}+\frac{\partial S_{12}}{\partial y} e_{x x}=0
$$

$\frac{\partial s_{12}}{\partial x}+\frac{\partial s_{22}}{\partial y}-\rho X_{\omega}+2 S_{12} \frac{\partial \omega}{\partial y}+\left(S_{11}-S_{22}\right) \frac{\partial \omega}{\partial x}$

$$
+\frac{\partial S_{22}}{\partial y} e_{x x}-\left(\frac{\partial S_{22}}{\partial x}+\frac{\partial S_{12}}{\partial y}\right) e_{x y}+\frac{\partial S_{12}}{\partial x} e_{y y}=0
$$

The strain components $e_{x x}, e_{y y}, e_{x y}$ and the rotation $\omega$ are defined as in Section 2. The body force components $X$ and $Y$ per unit mass are assumed to be constant. ${ }^{3}$ The coordinate system is chosen clockwise.

Incremental forces acting at a boundary per unit initial area (that is, per unit area as measured in the state of initial stress) are represented by the following $x$ and $y$ components

$$
\begin{aligned}
& \Delta f_{x}=\left(s_{11}-S_{12} \omega+S_{11} e_{y y}-S_{12} e_{x y}\right) \cos (n, x) \\
& +\left(s_{12}-S_{22} \omega-S_{11} e_{x y}+S_{12} e_{x x}\right) \cos (n, y) \\
& \Delta f_{y}=\left(s_{12}+S_{11 \omega}+S_{12} e_{y y}-S_{22} e_{x y}\right) \cos (n, x) \\
& +\left(s_{22}+S_{12} \omega-S_{12} e_{x y}+S_{22} e_{x x}\right) \cos (n, y) .
\end{aligned}
$$

These forces are acting on a closed contour in the $x, y$ plane and the normal direction chosen positive outward is designated by $n$.

\section{REFERENCES}

(1) M. A. Brot, "Theory of Elasticity with Large Displacements and Rotations," Proc. 5th Internat. Cong. Applied Mechanics, pp. 117-122 (1938).

(2) M. A. Biot, "Non-Linear Theory of Elasticity and the Linearized Case for a Body Under Initial Stress," Phil. Mag., Vol. 27, pp. 468-489 (1939).

(3) M. A. Bıот, "Elastizităts Theorie Zweiter Ordnung mit Anwendungen," Zeil. Ang. Math. und Mech., bd. 20, pp. 89-99 (1940).

(4) M. A. Brot, "Folding of a Layered Viscoelastic Medium Derived from an Exact Stability Theory of a Continuum Under Initial Stress," Quart. Appl. Math., Vol. XVII, pp. 185-204 (1959).

(5) M. A. BIот, "Instability of a Continuously Inhomogeneous Viscoelastic Half Space Under Initial Stress," Jour. FrankLin Inst., Vol. 270, pp. 190-201 (1960).

(6) M. A. BIot and H. OdÉ, "On the Folding of a Viscoelastic Medium with Adhering Layer Under Compressive Initial Stress," Quart. Appl. Math., Vol. XIX, pp. 351-355 (1962).

(7) M. A. Bгот, “Surface Instability of Rubber in Compression," Appl. Sci. Res. A, 12 (1963). (On press.)

(8) M. $\Lambda$. Bıot, "Exact Theory of Buckling of a Thick Slab," Appl. Sci. Res. $\Lambda, 12$ (1963') (On press.)

(9) M. A. Brot, "The Influence of Initial Stress on Elastic Waves," J. Appl. Phys., Vol. 11, pp. 522-530 (1940).

(10) M. A. Biot, "Internal Buckling Under Initial Stress in Finite Elasticity," Proc. Roy. Soc., Ser. A, Vol. 273, No. 1354, pp. 306-328 (1963).

(11) M. A. Bıот, "Surface Instability in Finite Anisotropic Elasticity Under Initial Stress," Proc. Roy. Soc., Ser. A, Vol. 273, No. 1354, pp. 329-339 (1963).

(12) M. A. Bıor, "Interfacial Instability in Finite Elasticity Under Initial Stress," Proc. Roy. Soc., Ser. A, Vol. 273, No. 1354, pp. 340-344 (1963).

(13) M. A. Biot, "Incremental Elastic Coefficients of an Isotropic Medium in Finite Strain," App. Sci. Res., A, 12 (1963). (On press.)

(14) A. N. Haskell, "Dispersion of Surface Waves in Multilayered Media," Bull. Seism. Soc. $A m .$, Vol. 43, p. 17 (1953).

3 The more general equations are found in $(1,2,3,7)$. 
polnote page 136

Sonations (80)

$(81)$

oolven of $a_{12}$ and $b_{12}$ $\beta^{2}$ sould be mullayg outside prat 